\title{
Photorefractive phase coupling measurement using self-stabilized recording technique
}

\author{
R. Montenegro*, I. de Oliveira**, A.A. Freschi ${ }^{\dagger}$ and J. Frejlich* \\ * Laboratório de Óptica - IFGW - UNICAMP - Campinas-SP, Brazil \\ ** CESET-UNICAMP, Limeira-SP, Brazil \\ ${ }^{\dagger}$ Departamento. de Física - IGCE - UNESP - Rio Claro-SP, Brazil
}

\begin{abstract}
The measurement of the phase shift $\varphi$ between the transmited and difracted beams interfering along the same direction behind the hologram recorded in a photorefractive crystal is directly and accurately measured using a self-stabilized recording technique. The measured phase shift as a function of the applied electric field allows computing the Debye sereening lenght and the effectively applied field coefficient of an undoped $\mathrm{Bi}_{12} \mathrm{TiO}_{20}$ crystal. The result is in good agreement with the already available information about this sample.
\end{abstract}

\section{INTRODUCTION}

The interference pattern of light projected onto a photorefractive material and the resulting volume hologram are in general phase shifted by a quantity $\phi$ which on its turn produces a phase shift $\varphi$ between the transmitted and the diffracted beams behind the photorefractive crystal. Such phase shift provide with relevant information about the nature of the recording mechanism and the sample under analysis [1,2]. The phase shift $\varphi$ is easier to measure than $\phi$ but in any case the measurement is always very noisy because of the high sensitivity of holographic setups to environmental perturbations. It is nevertheless possible to considerably reduce such perturbations by using self-stabilized recording techniques $[2,3]$. Such a procedure imposes a value $\varphi=0, \pi$ or $\pm \pi / 2$ depending on the way stabilization is carried out. This $\varphi$ is certainly not the unconstrained value we want to measure. It is however possible to stabilize the recording pattern of fringes (and the holographic setup) on an external reference (for ex. the pattern of fringes produced by the transmitted and reflected beams on a small glass plate placed close to the sample under analysis) as already proposed elsewhere $[2,4]$. The advantage of this technique is that the stabilization does not affect the recording process on the photorefractive crystal sample so that the phase shift is free from constraints. The handicap is that stabilization on an external reference is far less effective than self-stabilization on the sample itself.

In this paper we propose to use self-stabilization with arbitrarily selected phase shift $[2,5]$ for the measurement of the unconstrainted value of $\varphi$. This technique is similar to the usual self-stabilization with the difference that, instead of imposing either $\varphi=0, \pi$ or $\varphi= \pm \pi / 2$, we are free to choose any value for $\varphi$ for stabilization. The details of this technique are described elsewhere $[2,5]$ and the important fact here is that if the selected value of $\varphi$ for stabilization is not the unconstrained one, the holographic recording is not matching and because of that the hologram is continuously being erased to be recorded somewhere else. The result is a continuously moving hologram which speed depends on the difference between the unconstrained and the imposed value of $\varphi$, besides other material and experimental parameters. Instead, if the selected value for $\varphi$ is the same as the unconstrained one, the hologram will be a stationary non running one and the whole recording process will be self-stabilized and the very stable setup will allow to accurately measure $\varphi$. It is very simple to detect whether the hologram is running or not so that the desired value of $\varphi$ can be easily found out.

\section{THEORY}

The overall irradiance along the direction $I_{S}$ behind the sample, the phase shift $\varphi$ and the diffraction efficiency are respectively described by the expressions $[6,7]$

$$
I_{S}=I_{S}^{o}(1-\eta)+I_{R}^{o} \eta \pm 2 \sqrt{\eta(1-\eta)} \sqrt{I_{S}^{o} I_{R}^{o}} \cos \varphi
$$




$$
\begin{array}{r}
\tan \varphi=\frac{\sin (\gamma d / 2)}{\frac{1-\beta^{2}}{1+\beta^{2}}(\cosh (\Gamma d / 2)-\cos (\gamma d / 2))+\sinh (\Gamma d / 2)} \\
\eta=\frac{2 \beta^{2}}{1+\beta^{2}} \frac{\cosh (\Gamma d / 2)-\cos (\gamma d / 2)}{\beta^{2} e^{-\Gamma d / 2}+e^{\Gamma d / 2}}
\end{array}
$$

where $I_{S}^{o}$ and $I_{R}^{o}$ are the input irradiances, $\beta^{2} \equiv I_{R}^{o} / I_{S}^{o}, d$ is the thickness of the sample, $\Gamma$ and $\gamma$ are:

$$
\begin{array}{rc}
\Gamma(x)=4 w E_{D} \frac{x^{2} K^{2} l_{s}^{2}+K^{2} l_{s}^{2}+1}{\left(1+K^{2} l_{s}^{2}\right)^{2}+K^{4} l_{s}^{4} x^{2}} \quad \gamma(x)=4 w \frac{E_{D} x}{\left(1+K^{2} l_{s}^{2}\right)^{2}+K^{4} l_{s}^{4} x^{2}} \\
w=\frac{\pi n^{3} r_{\mathrm{eff}}}{2 \lambda} & x \equiv \xi E_{o} / E_{D} \quad E_{D} \equiv K \frac{k_{B} T}{q}
\end{array}
$$

Here $n$ is the sample's refractive index, $r_{\text {eff }}$ is the effective eletro-optic coefficient, $\lambda$ is the wavelength of the recording beams, $E_{o}$ is the applied eletric field, $E_{D}$ the diffusion electric field, $\xi$ the effective-field coefficient [2], $l_{s}$ the Debye lenght and $K$ the hologram vector value. Equations 2 and 3 are implicit functions of $E_{o}$ where the unknown parameters are $l_{s}$ and $\xi$ that can be found out form the theoretical fitting to the corresponding experimental data. The more accurate $\varphi$ is measured the more accurate value for $l_{s}$ is obtained. That is why stabilized recording is important here.

\section{EXPERIMENT}

The experimental setup is shown in Fig. 1 and the experiment consists in recording a hologram in self-stabilized form with adjustable $\varphi[5]$ with the difference that here $\varphi$ is selected so as to produce a stationary (non moving) hologram. The $\varphi$ value fullfiling this condition is the unconstrained one we are looking for. We find out the experimental $\varphi$ vs $E_{o}$ in this way on an undoped $\mathrm{Bi}_{12} \mathrm{TiO}_{20}$ crystal (labeled BTO-013), produced in Brazil, with dimensions: $d=2.35 \mathrm{~mm}$, inter-electrode distance $l=6.95 \mathrm{~mm}$ and $h=10.25 \mathrm{~mm}$ height. The index of refraction and the effective eletro-optic coefficient are $n \approx 2.6$ and $r_{\text {eff }}=5.610^{-12}$, respectively. Recording is carried out using the $514.5 \mathrm{~nm}$ wavelength laser line with $\beta^{2} \approx 10$ and $K=9.3 \mu \mathrm{m}^{-1}$. The holographic pattern of fringes is projected onto the (110) input crystal plane with the [001]-axis parallel to the crystal dimension $h$ and perpendicular to the hologram vector $\vec{K}$. The input beams polarization direction is chosen so that the diffracted and the transmitted beams behind the crystal are parallel polarized $[2,8]$. One of the recording beams is sinusoidally phase-modulated with frequency $\Omega /(2 \pi)=1.6 \mathrm{kHz}$ and phase amplitude $\psi_{D}=0.45 \mathrm{rad}$, using a PZT-supported mirror, in order to produce the first and second harmonics signals $V_{S}^{\Omega}$ and $V_{S}^{2 \Omega}$, respectively

$$
\begin{array}{r}
V_{S}^{\Omega}=A_{1} J_{1}\left(\psi_{D}\right) \sqrt{I_{S}^{o} I_{R}^{o}} \sqrt{\eta(1-\eta)} \sin (\varphi) \sin (\Omega t) \\
V_{S}^{2 \Omega}=A_{2} J_{2}\left(\psi_{D}\right) \sqrt{I_{S}^{o} I_{R}^{o}} \sqrt{\eta(1-\eta)} \cos (\varphi) \cos (2 \Omega t)
\end{array}
$$

as measured in the overall irradiance behind the sample, where $A_{1,2}$ is the overall amplification that depends on the experimental settings and $J_{n}$ is the Bessel function of order $n$. The same mirror used for phase modulation is used to operate the feedback stabilization loop. Silver-painted glue electrodes are used for applying the transverse electric field $E_{o}$ to the sample. The second harmonic $V_{S}^{2 \Omega}$ is fed to one input of a two-phase $2 \Omega$-tuned lock-in amplifier. The first harmonic $V_{S}^{\Omega}$ is frequency-doubled, amplified and phase-shifted to produce a new signal which we shall label $V_{S}^{\Omega 2}$

$$
V_{S}^{\Omega 2}=A_{2} J_{2}\left(\psi_{D}\right) \sqrt{I_{S}^{o} I_{R}^{o}} \sqrt{\eta(1-\eta)} \sin (\varphi) \sin (2 \Omega t)
$$

to distinguish it from the direct second harmonic. This signal is fed to the other lock-in amplifier input. The signals fed to both amplifier inputs are added and two demodulated signals result

$$
\begin{aligned}
& V_{X}=2 \sqrt{2} J_{2}\left(\psi_{D}\right) \sqrt{I_{S}^{o} I_{R}^{o}} \sqrt{\eta(1-\eta)} \sin \left(\varphi-\theta_{s}\right) \\
& V_{Y}=2 \sqrt{2} J_{2}\left(\psi_{D}\right) \sqrt{I_{S}^{o} I_{R}^{o}} \sqrt{\eta(1-\eta)} \cos \left(\varphi-\theta_{s}\right)
\end{aligned}
$$

where $\theta_{s}$ the reference phase set in the lock-in amplifier. The signal $V_{X}$ is chosen as error signal, imposing $\varphi=\theta_{s}$ and consequently $\cos \left(\varphi-\theta_{s}\right)=1$ and $V_{Y}$ becomes maximum. 


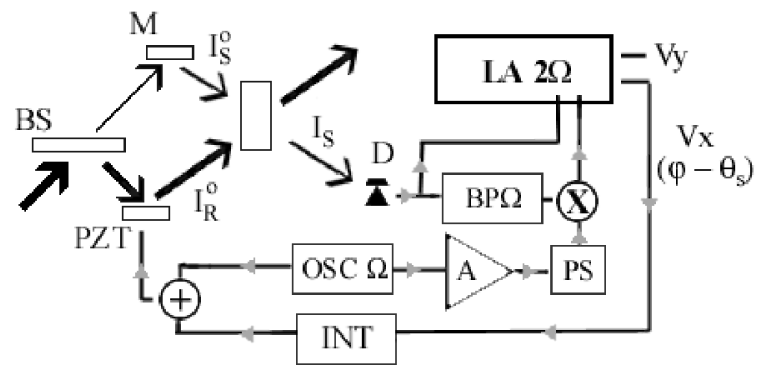

FIGURE 1. Experimental setup: PZT: pizo-electric supported mirror moving at frequency $\Omega, \mathrm{D}$ : photodetector, BP: band-pass filter $(\Omega)$, OSC: oscilator, A: amplifier, PS: phase shifter, INT: integrator, M: mirror, LA: Lock-in Amplifier tuned to $2 \Omega$.

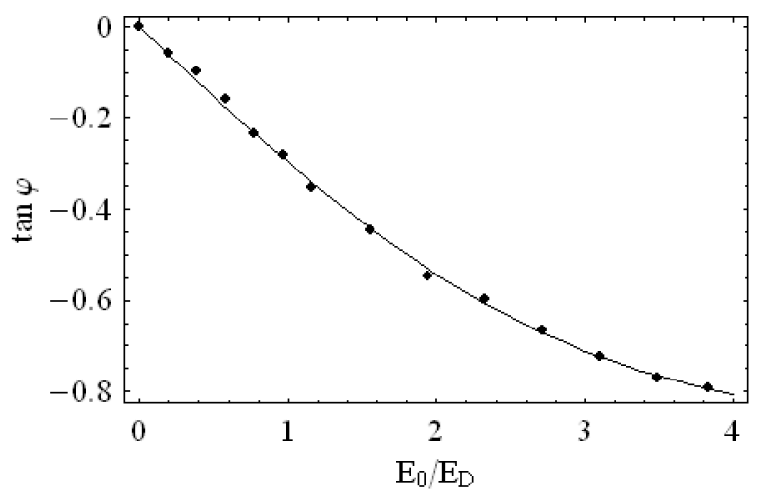

FIGURE 2. Equilibrium phase $(\tan \varphi)$ as function of the applied eletrical field $\left(E_{0}\right)$ for BTO-013 sample. The solid curve is the best theorical fit that leads to $\xi \approx 0.4$ and $l_{s}=0.068 \mu \mathrm{m}$.

\section{RESULTS AND DISCUSSIONS}

In stationary conditions the mathematical relation between $\varphi, \eta, \Gamma, \gamma$ and the applied field are quite simple mainly because it is not necessary to consider a varying response time along the sample thickness due to the exponential distribution of light. In this case experimental data analysis is considerably simplified. The following measurements were carried out on our BTO sample:

- Phase shift: By self-stabilized recording a hologram using $V_{X}$ as error signal with $\theta_{s}=\varphi$ such that a stationary hologram is produced, we computed the unconstrained $\varphi$ and plotted $\tan \varphi$ as a function of the applied $E_{o}$ as shown in Fig.2. Fitting Eq. 2 with Eqs.4-5 to these data we computed $l_{s}=0.068 \mu \mathrm{m}$ and $\xi=0.4$ for the sample under analysis.

- Harmonic $V_{Y}$ : Once the self-stabilized hologram above is achieved, the corresponding value of $V_{Y}$ is measured and $\eta$ is computed from Eq. 10 where $\cos \left(\varphi-\theta_{s}\right)=1$ and all other parameters in this equation being known. In this way it is possible to plot $\eta$ as a function of $E_{o}$ as shown in Fig.3. Fitting Eq.3 with Eqs.4-5 to these data allows also computing $l_{s}=0.056 \mu \mathrm{m}$ and $\xi=0.66$.

- Energy exchange: Diffraction efficiency can be also obtained from energy transfer considerations. In fact, once the hologram has been recorded, and at the moment beam $I_{R}^{o}$ is suddenly shut off, Eq.1 simplifies to

$$
\left(I_{S}\right)_{I_{R}^{o}=0}=I_{S}^{o}(1-\eta)
$$

and from the expression in Eq. 1 we obtain

$$
\frac{I_{S}-\left(I_{S}\right)_{I_{R}^{o}=0}}{\left(I_{S}\right)_{I_{R}^{o}}}=\beta^{2} \frac{\eta}{1-\eta} \mp \beta \sqrt{\eta} \approx \beta^{2} \eta \mp \beta \sqrt{\eta} \quad \eta \ll 1
$$

that allows computing $\eta$ once $\beta$ is known. Note that here one single beam measurement is involved and only a photodetector with linear response is requied. Once $\eta$ is computed in this way as a function of $E_{o}$ from a self-stabilized stationary hologram, as represented in Fig.4, we should proceed as in the item above to compute the parameters that turned out to be $l_{s}=0.065 \mu \mathrm{m}$ and $\xi=0.55$.

Our results show that the parametres (mainly $l_{s}$ ) computed from the phase shift and energy exchange are in reasonably good mutual agreement (less that $\pm 3 \%$ difference) whereas the value computed from the $V_{Y}$ is sensibly different. On the other hand experimental data from energy exchange (see Fig.4) are far more dispersed than observed in the other two experiments. This is due to the fact that energy exchange in sillenites is rather small and its measurement is therefore subject to large uncertainty. The $V_{Y}$-term technique instead can be measured with high accuracy but the value of the experimental parameters here involved ( $P Z T$ linearity and response, phase modulation $\psi_{D}$, for example) are difficult to be exactly evaluated, so that data are not much dispersed but the derived $l_{s}$ my be affected by a sistematic 


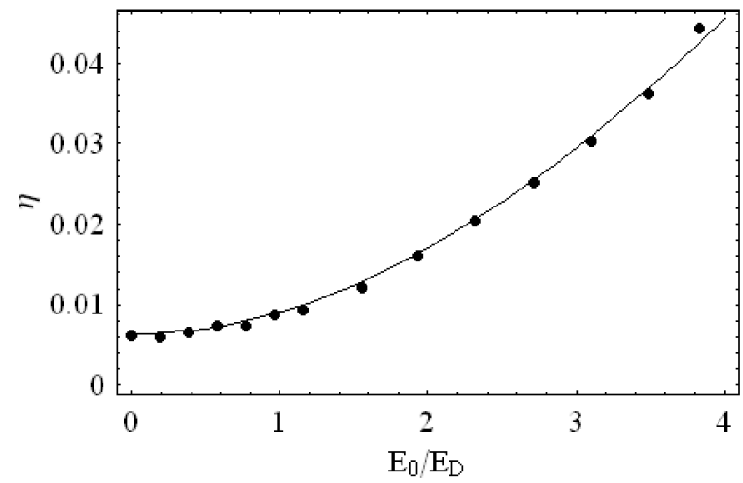

FIGURE 3. Difraction efficiency $(\eta)$ as function of the applied eletrical field $\left(E_{o}\right)$ calculated by harmonic $V_{Y}$. The solid curves were obtained using the best fitting parameters that lead to $\xi=0.66$ and $l_{s}=0.055 \mu \mathrm{m}$.

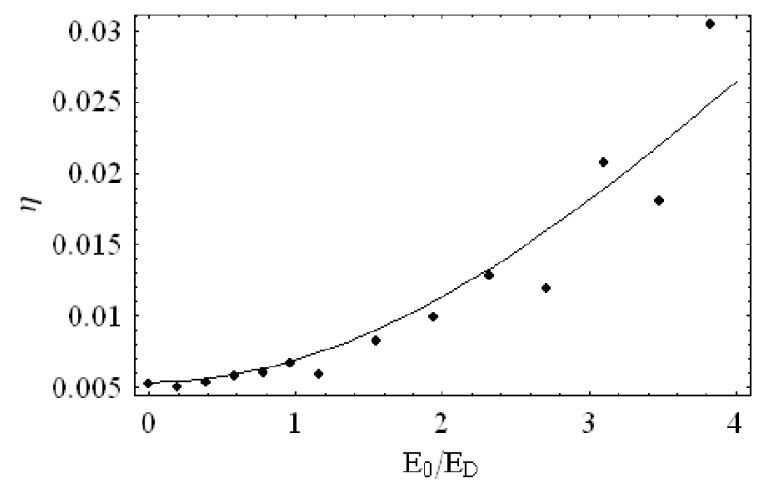

FIGURE 4. Difraction efficiency $(\eta)$ as function of the applied eletrical field $\left(E_{0}\right)$ calculated by energy exchange. The solid curves were obtained using the best fitting parameters that lead to $\xi=0.55$ and $l_{s}=0.065 \mu \mathrm{m}$.

error.

The mathematical model here involved is a very simple one just depending on one single material $\left(l_{s}\right)$ and one experimental parameter to be found out from fitting to experimetal data. A simple equation to fit and a lower number of parameters to be fitted certainly contribute to improve the reliability of the result. That is why we believe that the result here obtained $\left(l_{s}=0.068 \mu \mathrm{m}\right)$ from the direct phase shift technique is highly reliable in spite of being sensibly higher compared to those already reported for the same or similar samples using other techniques like the inicial phase $\operatorname{shift}\left(l_{s}=0.023 \mu \mathrm{m}\right)[6]$, running hologram [9] $\left(l_{s}=0.036 \mu \mathrm{m}\right)$ and fringed-locked hologram [10] $\left(l_{s}=0.048 \mu \mathrm{m}\right)$.

\section{CONCLUSIONS}

We have reported the successful self-stabilized recording of stationary (non moving) photorefractive holograms with arbitrary and unknown hologram phase shift. We have applied this technique, for the first time to our knowledge, for accurately finding out the phase shift and using these data for material characterization. The mathematical description of stationary photorefractive holograms is much simpler than for nonstationary ones and therefore the fitting of data is much simpler and more reliable too.

\section{ACKNOWLEDGMENTS}

The autors are grateful to the Conselho Nacional de Desenvolvimento Científico e Tecnlógico (CNPq), the Coordenação de Aperfeiçoamento de Pessoal de Nível Superior (CAPES) and Fundação de Amparo à Pesquisa do Estado de São Paulo (FAPESP) for the financial support.

\section{REFERENCES}

[1] M. G. Moharan, T. K. Gaylord, R. Magnusson, and L. Young, J. Appl. Phys. 50, 5642-5651 (1979).

[2] J. Frejlich, "Photorefractive Materials: Fundamental Concepts, Holographic Recording, and Materials Characterization”, Wiley-Interscience (New York), 2006.

[3] A. A. Kamshilin, J. Frejlich, and L. Cescato, Appl. Opt. 25, 2375-2381 (1986).

[4] A. A. Freschi, P. M. Garcia, and J. Frejlich, Appl. Phys. Lett. 71, 2427-2429 (1997).

[5] A. A. Freschi, and J. Frejlich, Opt. Lett. 20, 635-637 (1995).

[6] J. Frejlich, P. M. Garcia, K. H. Ringhofer, and E. Shamonina, J. Opt. Soc. Am. B 14, $1741-1749$ (1997).

[7] N. V. Kukhtarev, V. B. Markov, S. G. Odulov, M. S. Soskin, and V. L. Vinetskii, Ferroelectrics 22, $961-964$ (1979).

[8] A. A. Kamshilin and M. P. Petrov, Opt. Commun. 53, 23-26 (1985).

[9] I. de Oliveira, and J. Frejlich, J. Opt. Soc. Am. B 18, 291-297 (2001).

[10] M.C.Barbosa, L.Mosquera, and J. Frejlich, Appl. Phys. B 72, 717-721 (2001). 
Copyright of AIP Conference Proceedings is the property of American Institute of Physics and its content may not be copied or emailed to multiple sites or posted to a listserv without the copyright holder's express written permission. However, users may print, download, or email articles for individual use. 